\title{
Wavelet-based Image Enhancement Techniques for Improving Visual Quality of Ultrasonic Images
}

\author{
K. Karthikeyan \\ Assistant Professor, Department of Information Technology, \\ Dr. SNS Rajalakshmi College of Arts \& Science, \\ Coimbatore - 49, TN, India.
}

\author{
C. Chandrasekar \\ Associate Professor, Department of \\ Computer Science, Periyar University, \\ Salem - 11, TN, India.
}

\begin{abstract}
Medical imaging is concerned with the development of the imaging devices that help to identify different aspects of the tissue and organs based on various properties and reveal new properties of the tissue and internal structure. Ultrasonic devices are frequently used for medical imaging and the images produced by these devices often have to be converted to a form that is better suited for image analysis and understanding, which are referred as 'image enhancement techniques'. In this paper, three techniques are proposed for edge enhancement, image enlargement and image fusion. All the algorithms have the common goal of improving the visual quality of ultrasonic images and are based wavelets and other image processing techniques. The proposed models were tested vigorously using various test images obtained and the experimental results proved that the proposed models produce significant improvement over the existing traditional systems.
\end{abstract}

Keywords :

Anisotropic Diffusion, Image Fusion, Interpolation, BayesShrink, Fourth Order PDE, Speckle denoising, Wavelet.

\section{INTRODUCTION}

Medical imaging is concerned with the development of the imaging devices that help to identify different aspects of the tissue and organs based on various properties and reveal new properties of the tissue and internal structure. Medical image processing is a field of science that is gaining wide acceptance in healthcare industry due to its technological advances and software breakthroughs. It plays a vital role in disease diagnosis and in improved patient care. It also helps medical practitioners during decision making with regard to the type of treatment. Several state-of-the-art equipments produce human organs in digital form which includes X-ray-based devices, Computed Tomography (CT), Magnetic Resonance Imaging (MRI), Ultrasound (US), Positron Emission Tomography (PET) and Single Photon Emission Computed Tomography (SPECT). Irrespective of the equipment used, the raw images produced by such devices have to be processed so that the visual appearance of the image has to be converted to a form which is better suited for image analysis and understanding. The techniques and methods used for this purpose are often termed as 'Image Enhancement Techniques' and are widely used in research field. These techniques aid in the improvement of an image appearance by enhancing image features or by decreasing ambiguity between different regions of the image [2], [17].

In image processing, an acquired image can be improved by contrast adjustments, sharpening, deblurring and removal of noise. Among them, image denoising, edge enhancement, image fusion and image zooming are the frequently used techniques. Image noise is defined as the random variation of brightness or color information in images produced by medical devices or scanners. The presence of noise gives an image a mottled, grainy, textured or snowy appearance. Image denoising is a technique used to remove these unwanted pixels that obscure important parts. Different hybrid denoising models that combines anisotropic diffusion and wavelets for removing noise in ultrasonic medical images were investigated [8]. This paper enhances the working of anisotropic diffusion and proposes different wavelet shrinkage models to remove noise. The rest of the three techniques are dealt in this paper. Eventhough the techniques discussed can be used to enhance all types of medical images this paper uses ultrasonic images.

Ultrasonic images are created using diagnostic sonography or ultrasonography, which is an ultrasound-based diagnostic imaging technique and are used to visualize subcutaneous body structures including tendons, muscles, joints, blood vessels and internal organs for possible pathology or lesions. Obstetric sonography is commonly used during pregnancy and is widely recognized by the public [24]. Medical sonography is used in the fields of cardiology, gastroenterology, gynecology, neurology, obstetrics, urology and cardiovascular systems [21]. Images produced by these devices can be displayed, captured and broadcast through a computer using a frame grabber to capture and digitize the analog video signal [6], [7]. It is widely used by practitioners as they have no known long-term side effects and has the added advantage that it is non-intrusive to the patients [4]. The device provides live images, where the operator can select the most useful section for diagnosing and thus facilitating quick diagnoses [19].

The quality of the ultrasonic image can be improved through the use of edge enhancement, image fusion and interpolation techniques. Edge enhancement is used to improve the quality of image edges so as to help in other image processing applications like segmentation and organ recognition. An edge in an image is normally defined as the transition in the intensity of that image. Edge enhancement enhances the local discontinuities at the boundaries of different objects (edges) in the image. The second selected task, image fusion, is another novel method used for creating quality image. During acquisition, often more than one image from the same organ of the same person is created. These images can be analyzed and the best features of each image can be combined to form a new quality improved image. This process of combining features to reconstruct a new improved version of the image is called image fusion. The third selected task is image interpolation. Often doctors enlarge or zoom an image to view details of a portion in a higher image resolution. When an 
image is zoomed, blurring artifacts occurs and the quality of image degrades. Image interpolation techniques are used to improve an image in these conditions.

The paper is organized as follows, Section 2 provides a brief literature study on the selected three areas. Section 3 presents the methodology used to enhance medical images, while Section 4 presents the results of various experiments conducted. Section 5 concludes the work with future research directions.

\section{PREVIOUS STUDIES}

The studies related to the present topic of interest are discussed in this section.

\subsection{Edge Enhancement}

In literature, there are many attempts to enhance edges of medical images. To shift globally the intensity value in the approximation-coefficients to achieve contrast enhancement [1]. The drawback of the histogram equivalization procedure in the spatial domain was analyzed and proposed a waveletbased edge contrast enhancement method [2]. A wavelet transform domain filter, based on the LLMMSE filter to suppress noise and to enhance edges was investigated [18]. Wavelet phase filter used by [25] at finer scales in the wavelet domain to reduce noise, and a semi-soft wavelet shrinkage technique at coarse scales in the wavelet domain to further reduce noise.

\subsection{Image Fusion}

Image fusion is the combination of complementary information and redundant information through different imaging sensors in order to obtain more comprehensive and accurate image description. Pixel-based techniques are the most frequently used technique [5], [14], [16]. A number of region-based fusion schemes have been proposed [12], [15]. Wavelet transforms have been successfully used in many fusion schemes. A common wavelet analysis technique used for fusion is the discrete wavelet transform (DWT). It has been found to have some advantages over pyramid schemes such as: increased directional information; no blocking artifacts that often occur in pyramid-fused images; better signal-to-noise ratios than pyramid-based fusion; improved perception over pyramid-based fused images, compared using human analysis [9], [23].

\subsection{Image Interpolation}

The adaptive interpolation schemes were adopted by spatially adapts the interpolators to better match the local structure around the edges [1]. The isophote-oriented approach solves the ensuing partial differential equations (PDEs) to minimize the curvature of interpolated isophotes to reduce the zigzagging artifacts [13]. Based on the assumption that the image to be enhanced is the low pass filtered subband of a wavelet-transformed high-resolution image, wavelet-based approaches have also been proposed for image interpolation [3], [20]. Since human eyes are more sensitive to the edge areas than the smooth areas within an image, many algorithms have been proposed to improve the subjectively visual quality of edge regions in the images that need interpolation applied to them. The edge-directed method (NEDI) by [10] uses the covariance of the original image to estimate the covariance information of the high-resolution image. The bilinear method was modified by considering the direction information of edges to preserve better smoothing edges than traditional version [1], [22]. In order to improve the quality of interpolated images, neural network-based schemes [11], the minimum mean square-error estimation [26], and the autoregressive modeling [27] have also been proposed for adaptive filter design. From the literature study, it was found that eventhough several solutions for medical image enhancement have been proposed, the field still has not reached a maturity level. The current need is to have more techniques that can produce enhanced version of the original image and whose result can be used by the other advanced operations of the medical image processing systems.

\section{METHODOLOGY}

The image enhancement techniques proposed have the common objective of improving the visual quality of an ultrasonic image. The proposed framework, termed hereafter as Image Enhancement Framework (IEF), is given in Figure 1.

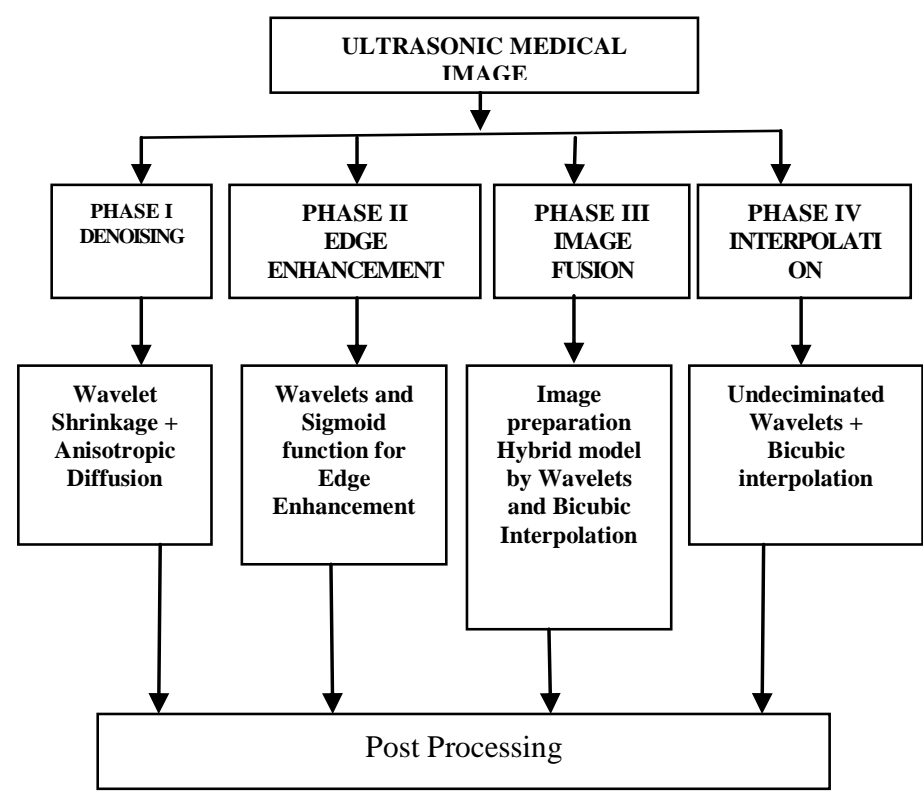

Figure 1 : Proposed Image Enhancement Framework (IEF)

All the proposed models employ the following three general steps.

- Decompose an image using wavelets.

- Apply selected enhancement algorithm.

- Perform inverse wavelet transform with the enhanced result to obtain a better enhanced image.

As mentioned earlier, the denoising part of IEF is dealt in detail and a review of previously published work is given in [7], [8].

\subsection{Edge Enhancement}

Edge enhancement is one of the most fundamental operations in image analysis. Edges form the outline of an object and an edge is the boundary between an object and the background. If the edges in an image can be identified accurately, all of the objects can be located and basic properties such as area, perimeter and shape can be measured. Edge detection, in general, consists of two parts: edge enhancement, which is a process for calculating the edge magnitude at each pixel; and edge localization, which is a process for determining the exact edge location. Once an edge is enhanced properly, the location of the edge can be identified accurately. Thus, the performance of edge detection depends on that of edge 
enhancement. Edge or border enhancement in ultrasound image is one of active research topics to improve the detectability of organ borders. Fixed size edge operators, like the gradient operations, always enhance both strong reflectors and speckle noise, spread the detected edges and decrease the contrast resolution of the image. Edge enhancement involves sharpening the outlines of objects and features with respect to their background.

In the proposed method, first the RGB image is converted into its YUV counterpart and the ' $\mathrm{Y}$ ' component alone is considered as they have more details regarding Human Visual System (HVS). Two dimensional wavelet decomposition is performed to obtain LL, LH, HL and $\mathrm{HH}$ subbands. It is known that the LL subband has the average details of the image, while LH contains horizontal edge details, HL has vertical edge details and $\mathrm{HH}$ subband elements contain diagonal edge details. Thus the detailed coefficients are selected. The edge enhancement procedure starts by dividing the wavelet into $8 \times 8$ blocks. The image features mean, variance and correlation are calculated for each block to obtain the local information in terms of texture pattern. Using this information the edges are categorized as strong and weak edges. The weak edges are then enhanced using a sigmoid function (Equation 1).

$$
y(x)=\frac{M}{1+e^{-\left(\frac{x-m-\Delta x}{a}\right)}+\Delta x}
$$

where $\mathrm{M}$ is $255, \mathrm{~m}=128$ (for 8 bit image), $\mathrm{x}$ is the edge pixel, $-127 \Delta \leq \mathrm{x} \leq+128$, parameter ' $\mathrm{a}$ ' refers to the speed of the change around the center. This process is repeated for detailed coefficients. Finally an inverse wavelet transformation is performed to obtain an edge enhanced image.

\subsection{Image Fusion}

Image fusion is a technique that integrates complementary information from multiple image data such that the new image is more suitable for processing tasks. An image can be described as collection of low or high band pass copies of an original image in which both the band limit and sample density are reduced in regular steps. In recent years, many solutions to image fusion have been proposed. All fusion algorithms have common objectives as given below,

- Preserve all relevant information in the fused image

- Suppress irrelevant parts of the image and noise

- Minimise any artifacts or inconsistencies in the fused image

In the proposed image fusion algorithm, Let the input source images be denoted as $S_{1}, S_{2}, \ldots, S_{n}$ and let $R$ be the final reconstructed image. Perform denoising and edge enhancement steps in a sequential manner to remove speckle noise and enhance the edges. Then a Content Sensitive Segmentation (CSS) is performed to separate the clear regions of each image, clear edges of each image and the blurring regions of each image. The CSS use the local contrast information for this purpose. The noise density of each clear part is calculated and the image with least noise is taken for fusion directly. Let this area be NE. Similarly, Figure of Merit (FoM) of each boundary area is calculated and the image with enhanced edge is taken for fusion. Let this area be CE. For the blurring region, DWT is performed and each subband is segmented using watershed algorithm. Using the energy of gradients, a Region Precedence Ranking (RPR) procedure is then used to arrange regions in precedence levels. Region based fusion rules that uses RPR are then constructed and used to selects a region from $S_{1}$ to $S_{2}$. Finally an inverse DWT is performed to combine the regions. Let this be WE. In the final step, NE, CE and WE are combined to construct the enhanced fused image.

\subsection{Image Interpolation}

When an ultrasonic image is given, it is often necessary to magnify details during analysis and diagnosis. Interpolation is the process where a small image is made larger by stretching the size of an image by generating pixels to fill in the blanks. The main concern here is to achieve interpolation without blurring or image artifacts, so as to make the enlarged image useful for analysis. Standard methods such as bilinear and bicubic spline interpolation tend to smooth out the edges since they do not utilize information relevant to preserving edge sharpness. Thus the main aim of the proposed interpolation technique is to maintain edge and detail information of an image while it is zoomed. For this purpose the bicubic method is combined with wavelets and region segmentation algorithm. The procedure adopted is briefly described below,

An image is initially divided in $\mathrm{K}$ blocks. For each block, the algorithm starts with a classification module which divides an image into edge and non-edge region, both of these regions are then enhanced using two separate procedures. The Edge Enhancement (EE) algorithm uses an angle evaluation module, a coordination rotation module and an interpolation module combines these results with bicubic method to enhance the edge region. The non-edge enhancement is performed using an undecimated DWT and quadtree weight function. The Undecimated Wavelet Transform (UWT) does not incorporate the downsampling operations. Thus, the approximation and detail coefficients at each level are the same length as the original signal. The UWT produces a denser approximation to the wavelet transform than the DWT approximation.

\section{EXPERIMENTAL RESULTS}

Several experiments were conducted to evaluate the proposed despeckling model. The performance metrics used are Peak Signal to Noise Ratio (PSNR) and Denoising Time. PSNR is a quality measurement between the original and a denoised image. The higher the PSNR the better the quality of the compressed or reconstructed image. To evaluate the proposed models the images in Figure 2 were used. All the proposed algorithms were developed using MATLAB 2009 and was tested on a Pentium IV machine with 2GB RAM.

\subsection{Edge Enhancement}

To analyze the performance of the proposed edge enhancement algorithm the Pratt's Figure of Merit (FoM) (Yu and Acton, 2002) is used and the results are shown in Table 1.

Table 1 : Figure of Merit

\begin{tabular}{|l|c|c|c|c|}
\hline & USI-1 & USI-2 & USI-3 & USI-4 \\
\hline Before & 0.74 & 0.75 & 0.75 & 0.74 \\
\hline After & 0.82 & 0.84 & 0.86 & 0.81 \\
\hline
\end{tabular}




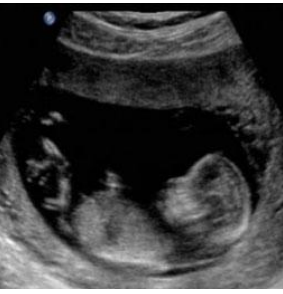

USI-1

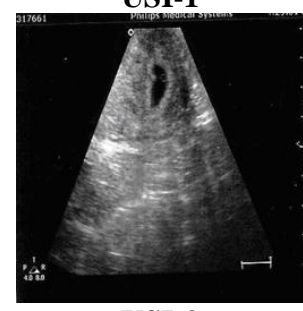

USI-3

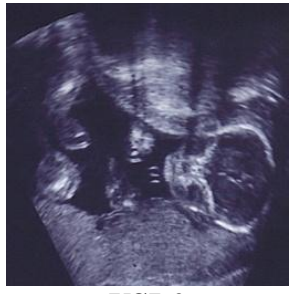

USI-2

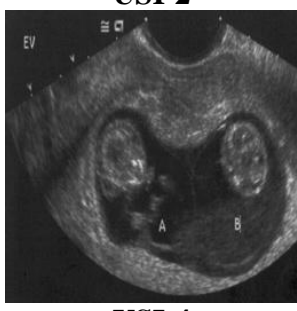

USI-4
Figure 2 : Test Images

By the higher nearing value to unity is achieved after applying the proposed model. It is clear that the proposed edge enhancement algorithm is successful in enhancing the edges while maintaining important image details. It can further be noticed that on an average the proposed algorithm showed an improvement of $10.51 \%$ when compared with the original image. Visual result of USI-3 before and after (enlarged to $200 \%$ ) is shown in Figure 3.

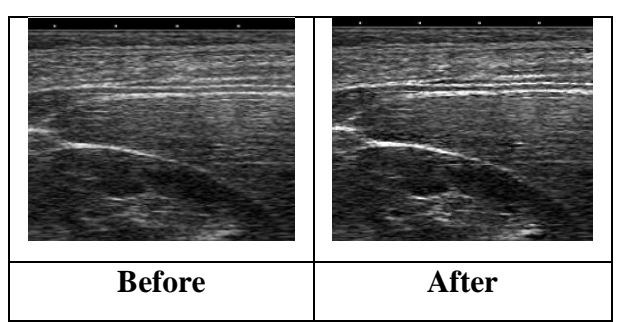

Figure 3 : Effect of the Proposed Edge Enhancer

Thus, the proposed edge enhancer, uses an adaptive segmentation based edge enhancement system and wavelets to identify edge details and enhanced the identified edges using sigmoid function. The results obtained showed that the proposed algorithm enhanced the edges in an efficient manner.

\subsection{Image Fusion}

The performance of the proposed image fusion algorithm is determined using Peak Signal to Noise Ratio and the results are compared with the traditional wavelet-based fusion algorithm. Analysis of the effect of noise removal algorithm and edge enhancement algorithm on image fusion is also analyzed. Table 2 shows the PSNR results.

TABLE 2 : PSNR of Image Fusion

\begin{tabular}{|l|c|}
\hline \multicolumn{1}{|c|}{ Algorithm Used } & $\begin{array}{c}\text { PSNR } \\
\text { (dB) }\end{array}$ \\
\hline Traditional Wavelets & 22.56 \\
\hline $\begin{array}{l}\text { Proposed algorithm without noise removal and } \\
\text { without edge enhancement }\end{array}$ & 24.94 \\
\hline $\begin{array}{l}\text { Proposed algorithm with noise removal and } \\
\text { without edge enhancement }\end{array}$ & 28.16 \\
\hline
\end{tabular}

\begin{tabular}{|l|c|}
\hline $\begin{array}{l}\text { Proposed algorithm without noise removal and } \\
\text { with edge enhancement }\end{array}$ & 23.17 \\
\hline $\begin{array}{l}\text { Proposed algorithm with noise removal and with } \\
\text { edge enhancement }\end{array}$ & 34.12 \\
\hline
\end{tabular}

From the table, it is clear that the proposed algorithm produce quality fusion image than the traditional wavelet algorithm. This is evident from the high PSNR values obtained. Moreover, from the results it is evident that the fusion process is further enhanced by the inclusion of the denoising and edge enhancement algorithms. From the experimental results obtained, it is clear that the proposed amalgamation of techniques improves the process of image fusion and produces image with high PSNR, better Edges and reduced artifacts.

\subsection{Image Interpolation}

The performance of the proposed image interpolation algorithm is determined using Peak Signal to Noise Ratio, FoM and Mean Structural Similarity (MSS). The results were compared with the traditional Bicubic and existing waveletbased algorithm (Figure 4).

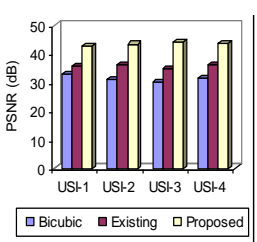

PSNR

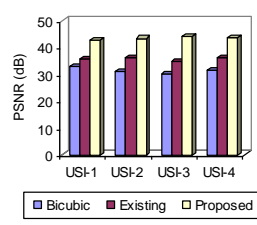

FoM

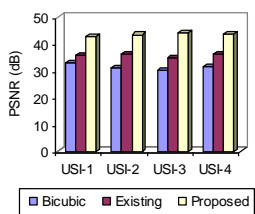

MSS
Figure 4 : Image Interpolation Results

The high PSNR value obtained shows that the proposed method produces an image which is very similar to the original one. Similarly, the near to unity value obtained with FoM and MSS show that the resultant image maintains edge and structure information of the original image in an efficient manner. Thus, the proposed interpolation method is efficient and the performance is favorable comparable with the existing techniques both in visual, edge and structural quality.

\section{CONCLUSION}

The present research work focus on developing image enhancement techniques designed specifically for ultrasonic images. In particular, four frequently used areas, namely, denoising, edge enhancement, enlargement and image fusion are considered. All the proposed models are constructed using wavelets and other image processing techniques. The experimental results proved that the proposed models produce significant improvement over the existing traditional systems.

\section{REFERENCES}

[1] Cha, Y. and Kim, S. (2007), The error-amended sharp edge (EASE) scheme for image, IEEE Transactions on Image Processing, Vol. 16, No. 6, pp. 1496-1606.

[2] Chanda, B. and Majumder, D.D. (2002), An edge preserving noise smoothing technique using multiscale morphology, Signal Processing, Elsevier North-Holland, Inc. Amsterdam, The Netherlands, Vol. 82, No. 4, pp. 527-544.

[3] Chang, S.G., Cvetkovic, Z. and Vetterli, M. (2006), Locally adaptive wavelet-based image interpolation, 
IEEE Transactions on Image Processing, Vol. 15, No. 6, pp. 479-482.

[4] Hangiandreou, N.J. (2003), Physics Tutorial for Residents: Topics in US: B-mode US: Basic Concepts and New Technology - Hangiandreou, Radiographics, Vol. 23, No.4, p. 1019.

[5] Hill, P. (2002), Wavelet Based Texture Analysis and Segmentation for Image Retrieval and Fusion, $\mathrm{PhD}$ thesis, Department of Electrical and Electronic Engineering, University of Bristol, UK.

[6] Karthikeyan, K. and Chandrasekar, C. (2010), Speckle Noise Reduction Techniques for Ultrasonic Image Enhancement - A Literature study", International Journal of Emerging Technologies and Applications in Engineering, Technology and Sciences (IJ-ETA-ETS), Vol. 3, Issue. 2, pp. 515-519.

[7] Karthikeyan, K. and Chandrasekar, C. (2010), A Study on the Application of Wavelets for Despeckling Ultrasound Images, International Journal of Computer Information Systems, Silicon Valley Publications, UK, Vol. 1, No. 5, pp: 48-54.

[8] Karthikeyan, K. and Chandrasekar, C. (2011), Speckle noise reduction of medical ultrasound images using Bayesshrink Wavelet threshold, International Journal of Computer Applications (IJCA, New York), Vol. 22, Number 9 .

[9] Li, H., Manjunath, S. and Mitra, S. (1995), Multisensor image fusion using the wavelet transform. Graphical Models and Image Processing, Vol. 57, No.3, pp.235245

[10] Li, X. and Orchard, M.T. (2001), New edge-directed interpolation, IEEE Transactions on Image Processing, Vol. 10, No. 10, pp. 1521-1527.

[11] Lin, C.T., Fan, K.W., Pu, H.C., Lu, S.M. and Liang, S.F. (2007), An HVS-directed neural-network-based image resolution enhancement scheme for image resizing, IEEE Transactions on Fuzzy Systems, Vol. 15, No. 4 pp. 605-615.

[12] Matuszewski, B., Shark, L.K. and Varley, M. (2000), Region-based wavelet fusion of ultrasonic, radiographic and shearographyc non-destructive testing images, Proceedings of the 15th World Conference on NonDestructive Testing, Rome.

[13] Morse, B.S. and Schwartzwald, D. (1998), Isophotebased interpolation, Proceedings of the IEEE International Conference on Image Processing, Chicago, Ill, USA, Vol. 3, pp. 227-231.

[14] Nikolov, S., Hill, P., Bull, D. and Canagarajah, N. (2001), Wavelets for image fusion. In A. Petrosian and
F. Meyer, editors, Wavelets in Signal and Image Analysis, Computational Imaging and Vision Series, Kluwer Academic Publishers, Dordrecht, The Netherlands, pp. 213-244.

[15] Piella, G. (2002), A region-based multiresolution image fusion algorithm. In ISIF Fusion 2002 conference, Annapolis.

[16] Piella, G. (2003), A general framework for multiresolution image fusion: from pixels to regions. Information Fusion, Vol.4, pp.259-280.

[17] Pratt, W.K. (2007), Digital Image Processing : PKIS Scientific Inside, 4th Edition, John Wiley \& Sons, Inc., New York.

[18] Reeves, T. and Jernigan, M. (1997), Multiscale-based Image enhancement, Canadian Conference on Electrical and Computer Engineering, Vol. 2, pp.500-505.

[19] Sudha, S., Suresh, G.R. and Sukanesh, R. (2009), Comparative Study on Speckle Noise Suppression Techniques for Ultrasound Images, International Journal of Engineering and Technology Vol. 1, No. 1, pp. 17938236.

[20] Temizel, A. and Vlachos, T. (2006), Wavelet domain image resolution enhancement, IEEE Proceedings Vision, Image and Signal Processing, Vol. 153, No. 1, pp. 25-30.

[21] Tso, B. and Mather, P. (2009), Classification Methods for Remotely Sensed Data (2nd ed.), CRC Press, pp. 37 38.

[22] Wang, Q. and Ward, R.K. (2007), A new orientationadaptive interpolation method, IEEE Transactions on Image Processing, Vol. 16, No. 4, pp. 889-900.

[23] Wilson, T., Rogers, S. and Kabrisky, M. (1995), Perceptual based hyperspectral image fusion using multi-spectral analysis. Optical Engineering, Vol.34, No.11, pp. 3154-3164.

[24] www.wikipedia.org

[25] Xu, K., Zheng, X. and Cheng, X. (1999), A novel method for image enhancement of medical images based on wavelet, Acta Electronica Sinica, Vol. 27, No. 9, pp. 121-123.

[26] Zhang, L. and Wu, X. (2006), An edge-guided image interpolation algorithm via directional filtering and data fusion, IEEE Transactions on Image Processing, Vol. 15 , No. 8, pp. 2226-2238

[27] Zhang, X. and Wu, X. (2008) Image interpolation by adaptive 2-D autoregressive modeling and soft-decision estimation, IEEE Transactions on Image Processing, Vol. 17, No. 6, pp. 887-896. 